\title{
Prognostic factors for survival in esophageal squamous cell carcinoma (ESCC) patients with a complete regression of the primary tumor (ypT0) after neoadjuvant chemoradiotherapy (NCRT) followed by surgery
}

\author{
Min Kong ${ }^{1,2,3}$, Jianfei Shen $^{2}$, Chao Zhou ${ }^{4}$, Haihua Yang ${ }^{4}$, Baofu Chen ${ }^{1,2,3}$, Chengchu Zhu ${ }^{2}$, Gongchao Wang ${ }^{1}$ \\ ${ }^{1}$ Department of Thoracic Surgery, Shandong Provincial Hospital, Cheeloo College of Medicine, Shandong University, Jinan, China; ${ }^{2}$ Department \\ of Cardiothoracic Surgery, Taizhou Hospital of Zhejiang Province Affiliated to Wenzhou Medical University, Taizhou, China; ${ }^{3}$ Department of \\ Cardiothoracic Surgery, Enze Hospital, Taizhou Enze Medical Center (Group), Taizhou, China; ${ }^{4}$ Department of Radiotherapy, Taizhou Hospital of \\ Zhejiang Province affiliated to Wenzhou Medical University, Taizhou, China \\ Contributions: (I) Conception and design: M Kong; (II) Administrative support: G Wang, C Zhu; (III) Provision of study materials or patients: \\ C Zhou, H Yang, B Chen; (IV) Collection and assembly of data: M Kong, J Shen; (V) Data analysis and interpretation: M Kong, J Shen; (VI) \\ Manuscript writing: All authors; (VII) Final approval of manuscript: All authors. \\ Correspondence to: Gongchao Wang. Department of Thoracic Surgery, Shandong Provincial Hospital, Cheeloo College of Medicine, Shandong \\ University, 9677 Jingshi Road, Jinan, China. Email: gongchao@sdu.edu.cn.
}

Background: There are also differences in survival prognosis among esophageal squamous cell carcinoma (ESCC) patients with a complete regression of the primary tumor (ypT0) after Neoadjuvant chemoradiotherapy (NCRT) followed by surgery. And the purpose of this study was to investigate influencing factors from these different prognostic outcomes and their possible causes.

Methods: The clinical data of 88 cases of ESCC patients with ypT0 after NCRT followed by surgery between 2011 and 2019 were retrospectively analyzed. The clinical and pathological prognostic factors that affect the survival were analyzed.

Results: Sex, number of lymph nodes dissected, and pathologic positivity of lymph nodes may be significant in univariate analysis $(\mathrm{P}<0.1)$. Further multivariate analysis suggested that the pathologic positivity of the lymph nodes was an independent factor affecting prognosis (HR: 4.757, 95\% CI: 2.195-10.313, $\mathrm{P}=0.000$ ). Subsequently, the whole group was divided into a positive lymph node group (group $\mathrm{LN}+$ ) and a negative lymph node group (group LN-) for comparison. The overall survival (OS) of group $\mathrm{LN}+$ was significantly worse (HR: 0.211, 95\% CI: 0.0336-0.239; $\mathrm{P}<0.0001$ ), and recurrence-free survival (RFS) was significantly poorer in the $\mathrm{LN}+$ group (HR: $0.0679,95 \% \mathrm{CI}: 0.0239-0.1923, \mathrm{P}<0.0001$ ). There were 14 cases of recurrence and metastasis in the $\mathrm{LN}+$ group $(14 / 21,66.7 \%)$ and 10 cases in the group LN(10/67, 14.9\%). Among the sites of recurrence and metastasis, there were $10(10 / 14,71.4 \%)$ and $4(4 / 14$, $28.6 \%$ ) cases of distant metastasis, respectively, and $4(4 / 14,28.6 \%)$ cases of local metastasis in the LN+ group; meanwhile, there were $8(8 / 10,80.0 \%)$ cases of distant metastasis and $2(2 / 10,20.0 \%)$ cases of local metastasis in the LN- group.

Conclusions: The independent risk factor for survival prognosis in ESCC patients with ypT0 after NCRT followed by surgery was positive postoperative pathological lymph nodes. The reason for the shortened survival time associated with this group of patients was their susceptibility to recurrence and metastasis.

Keywords: Esophageal squamous cell carcinoma (ESCC); neoadjuvant chemoradiation; esophagectomy; prognostic factors

Submitted May 21, 2020. Accepted for publication Aug 18, 2020.

doi: 10.21037/atm-20-4864

View this article at: http://dx.doi.org/10.21037/atm-20-4864 


\section{Introduction}

Esophageal cancer (EC) is one of the most common malignant tumors in the world and one of the six most fatal tumors $(1,2)$. Squamous cell carcinoma is the most common pathological type of EC. In 2012, there were about 398,000 new esophageal squamous cell carcinoma (ESCC) cases, accounting for $87 \%$ of EC (3).

Current EC treatment frequently involves multiple treatment modalities, including endoscopic resection (ER), esophagectomy, radiation therapy, chemotherapy (CT), chemoradiotherapy (CRT), immunotherapy (IT), and targeted therapy.

After surgery alone, the prognosis for patients with locally advanced EC remains poor, with a 5-year survival rate of only $25 \%$ (4). Recent evidence has suggested neoadjuvant chemoradiotherapy (NCRT) plus surgery may bring long-term survival benefits for locally advanced EC, especially $\operatorname{ESCC}(5,6)$.

A number of studies have confirmed that pathological complete response (PCR) remains one of the most important prognostic factors of long-term survival in EC after NCRT followed by surgery (7-10). PCR is defined as there being no evidence of residual tumor cells in the primary site and lymph nodes on operative specimens. However, in actuality, because the lymph nodes of EC often grow across regions, it is impossible to ensure that all the positive lymph nodes are removed during the operation; thus, PCR is only relative, and the condition of patients with a complete regression of primary tumor after NCRT with or without positive lymph nodes (ypT0) in the study seems to be closer to real-world experience.

There are also differences in survival prognosis among ESCC patients with ypT0 after NCRT followed by surgery. Thus, the purpose of this study was to investigate the influencing factors for these different prognostic outcomes and their possible causes.

There was a similar report in the PubMed (11), unlike their study, we used the same chemotherapy regimen and the same surgical procedure for each patient. Therefore, the novel point of view in our article is that even if the bias caused by different treatment schemes is avoided, the conclusion obtained is similar to the above study. We present the following article in accordance with the STROBE reporting checklist (available at http://dx.doi. org/10.21037/atm-20-4864).

\section{Methods}

\section{Data source and study population}

We reviewed the records of 151 consecutive patients with locally advanced ESCC who received NCRT followed by surgery treatment at the Enze Medical Center (group) of Taizhou city, Zhejiang province, China from January 2011 to June 2019. These data were extracted from our surgical database. According to the following inclusion criteria, a total of 88 cases were eligible for statistical analysis.

The criteria for inclusion were: (I) age between 18 and 70 years, (II) resectable thoracic ESCC clinically staged as T1-4N1M0/T4N0M0 (stage IIB or III) before treatment (12), (III) normal hematologic, renal, and hepatic function, and a Karnofsky performance score (KPS) of $\geq 90$, (IV) a complete regression of the primary tumor (ypT0) after NCRT followed by surgery, (V) no major perioperative complications, and (VI) complete follow-up data.

All procedures performed in this study involving human participants were in accordance with the Declaration of Helsinki (as revised in 2013). This study was approved by the ethics committee of Enze Hospital of the Taizhou Enze Medical Center (group) in Zhejiang province, China. As this was a retrospective study, informed consent was not required.

\section{Treatment plan}

\section{Preoperative chemotherapy regimen}

Vinorelbine $25 \mathrm{mg} / \mathrm{m}^{2}$ was given intravenously on day 1,8 , 22 , and 29 , and cisplatin $25 \mathrm{mg} / \mathrm{m}^{2}$ was given intravenously on day 1 to 4 and day 22 to 25 .

\section{Preoperative radiotherapy regimen}

Gross tumor volume (GTV) included primary esophageal tumor and metastatic lymph nodes, clinical target volume (CTV) included a subclinical lesion (normal esophagus of $3 \mathrm{~cm}$ above and below esophageal tumor) and corresponding paraesophageal lymphatic drainage area, and planned target volume (PTV) included CTV plus $8 \mathrm{~mm}$. A total dose of 40.0 Gy was administered in 20 fractions of 2.0 Gy, five fractions per week, starting on the first day of the first cycle of chemotherapy.

\section{Operation plan}

About 6 weeks after the end of chemoradiotherapy, a 
modified McKeown minimally invasive esophagectomy (13), including two-field lymphadenectomy with total mediastinal lymph node dissection, was performed.

\section{Variable collection and definition}

General clinical data that could have affected the prognosis were collected, including age, sex, body mass index (BMI), tumor location, clinical stage before treatment, cycles of chemotherapy, the interval between the end of NCRT and surgery, number of lymph nodes dissected, positive lymph nodes dissected, recurrence site, overall survival (OS) time, relapse-free survival (RFS), etc.

The histologic criteria for the response of NCRT was primarily based on postoperative pathology specimens and mainly considers the extent of tumor cell reduction, with a specific reference to the Owaki T. standard which defines the following grades (14): grade I, poor effect on radiation and chemotherapy with no significant change to tumors (necrosis or disappearance of the tumor is present in no more than two thirds of the whole lesion); grade II, tumor obviously changed by radiation and chemotherapy (necrosis or disappearance of the tumor is present in more than two thirds of the whole lesion, but viable tumor cells still remain); and grade III, pathological objective response consisting of complete disappearance of macroscopic and microscopic cancers, with or without the presence of granulation tissue. Esophageal grade primary site grade III was judged as a pathologic complete response of the primary tumor.

OS was defined as the time from date of surgery to death or loss to follow-up. RFS was defined as the time from the date of surgery to objective record of disease recurrence.

\section{Statistical methods}

For continuous variables, the number of observations $(\mathrm{N})$, the mean value, the minimum and maximum values, and the $95 \%$ confidence interval (CI) of descriptive statistical variables were calculated. $T$-test or a non-parametric test (Wilcoxon test, etc.) was used for the comparison between the two groups. For discontinuous variables (classification variables), descriptive statistics were used to calculate the number of cases and frequency (percentage) of each type. The two groups were compared using Pearson's chi-square test or Fisher's exact test. For the survival follow-up data, Kaplan-Meier analysis was used to estimate the median and $95 \%$ CI of OS and RFS, and to plot the survival curve. The differences in RFS and OS between the two groups were compared by using the log-rank method. A Cox proportional hazards risk regression model was used to compare the survival analysis and subgroup analysis of various prognostic factors affecting OS. A $\mathrm{P}$ value $<0.05$ was considered statistically significant.

A multivariate model was constructed by means of a stratified Cox regression analysis, using the variables in the univariate analysis that were found to be significant: variables with a $\mathrm{P}<0.1$ were included in a stepwise conditional forward model. All analyses were performed using IBM SPSS statistics software, version 24 (SPSS Inc., Chicago, IL, USA).

\section{Results}

\section{Overall baseline characteristics}

As shown in Table 1, 88 patients, including 72 (81.8\%) males and $16(18.2 \%)$ females, were enrolled, with an average age of 56 years (31-70 years), including $64(72.7 \%)$ patients aged 60 years or less and $24(27.3 \%)$ patients aged over 60 years. Thirteen $(14.8 \%)$ patients had a BMI of lower than $18.5 \mathrm{~kg} / \mathrm{m}^{2}, 53(60.2 \%)$ patients had a BMI of $18.5-23.9 \mathrm{~kg} / \mathrm{m}^{2}$, and $22(25.0 \%)$ patients had a BMI of more than $24 \mathrm{~kg} / \mathrm{m}^{2}$. Tumors were located in the proximal third, middle third, and distal third in 10, 65, and 13 cases, respectively. For pretreatment clinical stages, 15, 52, and 21 cases were T2, T3, and T4, respectively; 8 and 80 cases were N0 and N1, respectively; and 15 and 73 cases were stage IIB and III, respectively.

\section{Treatment-related prognostic indicators}

As shown in Table 2, 7 patients $(8.0 \%)$ completed 1 cycle of preoperative chemotherapy, and 81 patients $(92.0 \%)$ completed 2 cycles. The interval between the end of NCRT and surgery was $\leq 6$ weeks in 42 cases $(47.7 \%)$ and $>6$ weeks in 46 cases $(52.3 \%)$. The number of lymph nodes dissected was $>19$ in 48 cases $(54.5 \%)$ and $\leq 19$ in 40 cases (45.5\%). Postoperative pathological lymph nodes were negative in 67 cases $(76.1 \%)$ and positive in 21 cases (23.9\%).

\section{Univariate analysis and multivariate analysis}

Univariate analysis was conducted for age, sex, BMI, tumor location, clinical staging, and cycles of chemotherapy, the interval between the end of NCRT and surgery, number of 
Table 1 Patient characteristics ( $\mathrm{N}=88)$

\begin{tabular}{lc}
\hline Variable & Value \\
\hline Age at primary treatment (years) & $56[31-70]$ \\
$\leq 60 \mathrm{y}, \mathrm{n}(\%)$ & $64(72.7)$ \\
$>60 \mathrm{y}, \mathrm{n}(\%)$ & $24(27.3)$ \\
Sex, $\mathrm{n}(\%)$ & \\
Male & $72(81.8)$ \\
Female & $16(18.2)$ \\
BMI, $\mathrm{n}(\%)$ & \\
$<18.5 \mathrm{~kg} / \mathrm{m}^{2}$ & $13(14.8)$ \\
18.5-23.9 kg/m ${ }^{2}$ & $53(60.2)$ \\
$\geq 24 \mathrm{~kg} / \mathrm{m}^{2}$ & $22(25.0)$ \\
Tumor location, $\mathrm{n}(\%)$ & \\
Proximal third & $10(11.4)$ \\
Middle third & $65(73.9)$ \\
Distal third & $13(17.0)$ \\
Clinical T stage, $\mathrm{n}(\%)$ & $13(14.8)$ \\
cT2 & \\
cT3 & \\
III & \\
Nlinical N stage, $\mathrm{n}(\%)$ & \\
\hline
\end{tabular}

BMI, body mass index.

lymph nodes dissected, and positive lymph nodes dissected.

Multivariate Cox regression analysis included sex $(\mathrm{P}=0.072)$, number of dissected lymph nodes $(\mathrm{P}=0.027)$, and pathological positive lymph nodes $(\mathrm{P}=0.000)$, all elements which exhibited possible influences on prognosis in univariate analysis. Only pathologic lymph node positive was found to be an independent factor affecting prognosis (HR: 4.757,95\% CI: $2.195-10.313, \mathrm{P}=0.000)$, as shown in Table 3.

\section{Group analysis}

In the multivariate analysis, positive lymph node pathology
Table 2 Treatment-related factors $(\mathrm{N}=88)$

\begin{tabular}{lc}
\hline Characteristic & Value \\
\hline Cycles of chemotherapy, n (\%) & $7(8.0)$ \\
1 & $81(92.0)$ \\
2 & \\
Interval between the end of NCRT and & \\
surgery, n (\%) & $42(47.7)$ \\
$\leq 6$ weeks & $46(52.3)$ \\
$>6$ weeks & \\
Number of lymph nodes dissected, n (\%) & $48(54.5)$ \\
$>19$ & $40(45.5)$ \\
$\leq 19$ & \\
Positive lymph nodes dissected, n (\%) \\
No \\
Yes
\end{tabular}

was an independent prognostic factor affecting survival; therefore, the whole group was divided into a positive lymph node group (group $\mathrm{LN}_{+}$) and negative lymph node group (group LN-). Compared with the two groups, the OS of Group LN+ was significantly worse (HR: 0.211, 95\% CI: $0.0336-0.239 ; \mathrm{P}<0.0001$ ), as shown in Figure 1.

When RFS was compared between the two groups, the prognosis of the $\mathrm{LN}+$ group was poor and there was a significant difference (HR: 0.0679, 95\% CI: 0.0239-0.1923, $\mathrm{P}<0.0001$ ), as shown in Figure 2.

\section{Recurrence and metastasis analysis}

Among the 88 patients in the whole group, 24 (27.3\%) had recurrence and metastasis, 21 of whom $(21 / 24,87.5 \%)$ had RFS of less than 3 years, and the cumulative recurrence rate was positively correlated with mortality ( $\mathrm{rs}=0.979, \mathrm{P}<0.000$ ) (Figure 3). There were 14 cases of recurrence and metastasis in the LN+ group $(14 / 21,66.7 \%)$ and 10 cases in the LNgroup $(10 / 67,14.9 \%)$, with a significant difference between the two groups $(\mathrm{P}<0.000)$. There were 18 cases of distant metastasis (18/24, 75.0\%), 6 cases of local metastasis (6/24, $25.0 \%), 10$ cases of distant metastasis $(10 / 14,71.4 \%)$, and 4 cases of local metastasis $(4 / 14,28.6 \%)$ in the $\mathrm{LN}+$ group, and 8 cases of distant metastasis $(8 / 10,80.0 \%)$ and 2 cases of local metastasis $(2 / 10,20.0 \%)$ in the $\mathrm{LN}$ - group, as shown in Figure 4. 
Table 3 Univariate analysis and multivariate analysis of prognostic factors

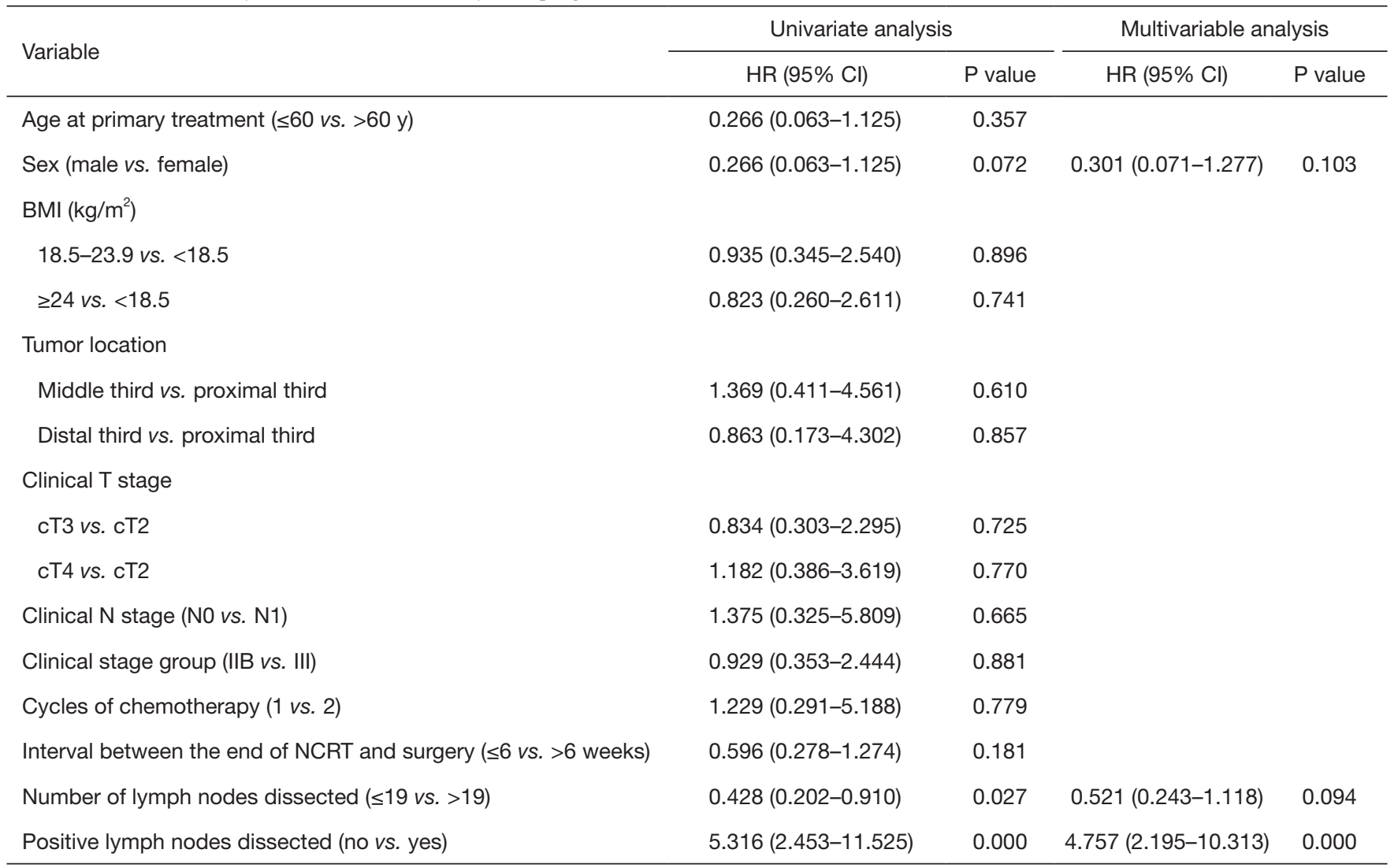

BMI, body mass index; NCRT, neoadjuvant chemoradiotherapy.

\section{Discussion}

This study found that positive postoperative lymph nodes were found to be an independent risk factor for the survival and prognosis of ESCC patients with ypT0 after NCRT followed by surgery. Positive postoperative lymph nodes were more likely to relapse and metastasize, and the survival time was shortened. However, there appears to be no statistical significance in the relationship between survival and classic prognostic factors including preoperative pathological $\mathrm{T}$ stage $(6,15)$. The findings in this study also underline the recommendations for neoadjuvant pathologic staging of cancer of the esophagus and esophagogastric junction for the American Joint Committee on Cancer (AJCC) and the International Union Against Cancer (UICC) (AJCC/UICC) 8th edition staging manuals indicating that $\mathrm{ypN}+$ portends poor survival irrespective of $\mathrm{yT}$ - stage (16).

Many studies have proven that NCRT combined with surgery can bring survival benefits to patients with EC, especially SCC $(5,6,17)$, especially for PCR cases with obvious advantages after chemoradiotherapy $(7,18)$. However, due to tumor heterogeneity, the response of primary lesions and lymph nodes to NRCT is not completely consistent, and there are cases of complete and partial pathological response after NCRT. Moreover, EC lymph nodes often grow cross-regionally, and intraoperatively, there is no guarantee that all positive lymph nodes can be removed, rendering PCR rather relative. However, primary focal pathologic mitigation after NCRT can be determined, so for the primary lesion patients with this condition, the situation aligns more with real-world experience. Thus, there remain many unknowns concerning the prognostic factors for survival in patients with ESCC with a PCR of the primary tumor after NCRT followed by surgery (19).

Recently, several studies have explored the prognostic value of pathologic response grades after NCRT in EC. Depypere's research (18) had similar results to our study, including the suggestion that ypT0N+ in EC patients following NCRT has a poor prognosis and behaves similar 


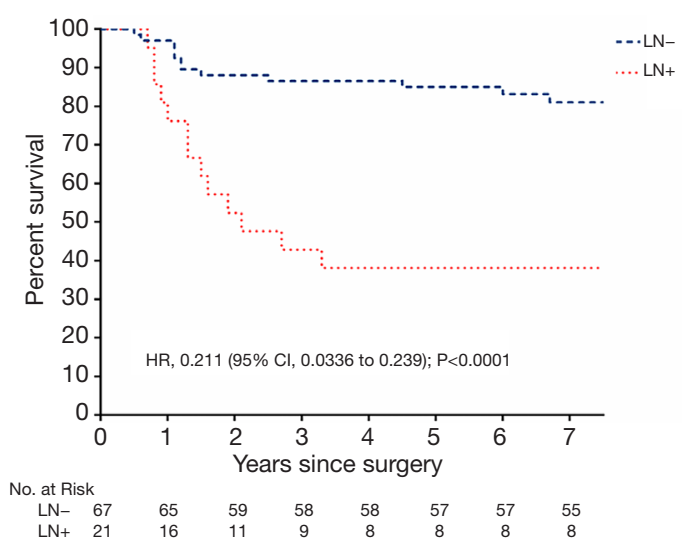

Figure 1 Overall survival (OS) in the $\mathrm{LN}+$ group and the LNgroup.

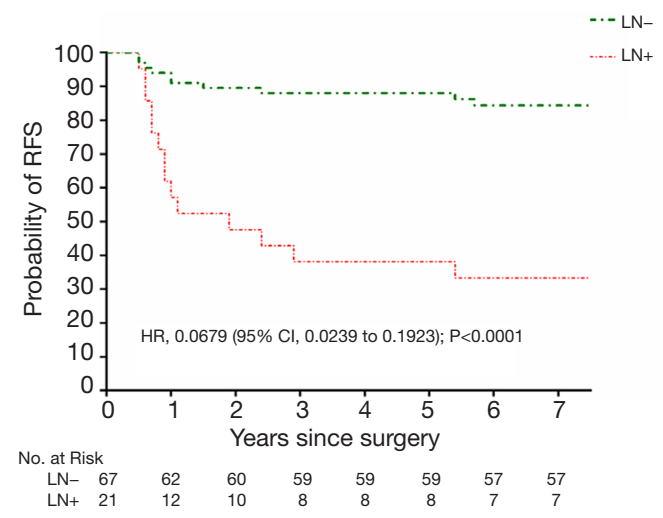

Figure 2 Recurrence-free survival (RFS) in the $\mathrm{LN}+$ group and LN- group.

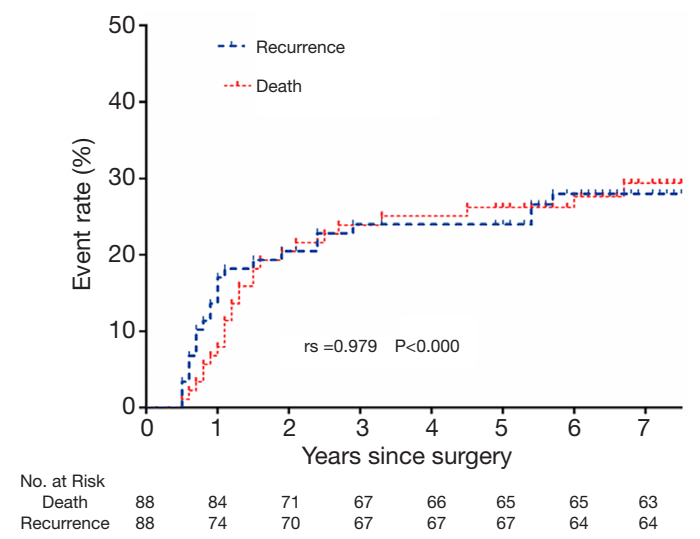

Figure 3 Relationship between cumulative recurrence rate and mortality.

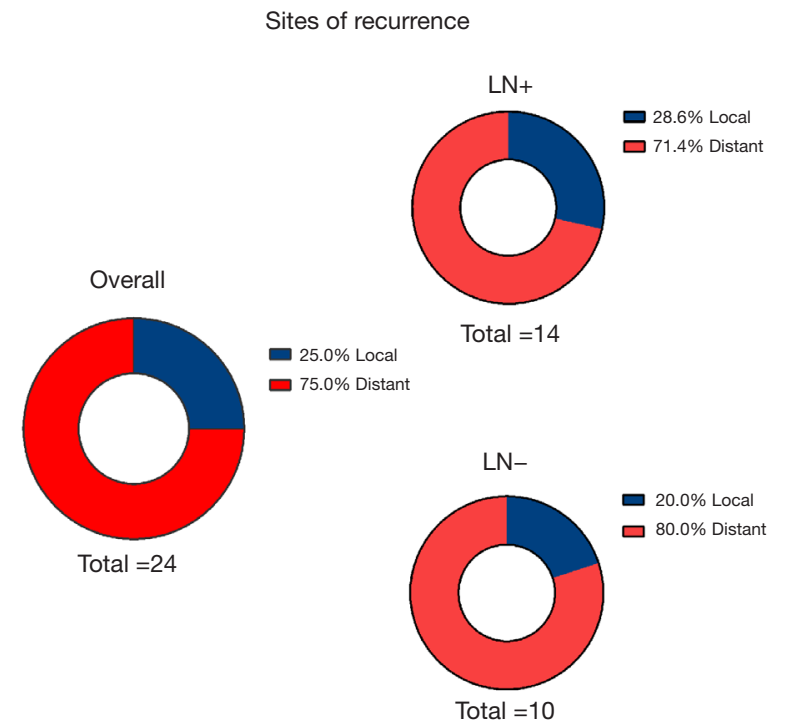

Figure 4 Distribution of recurrence sites.

to yp $\mathrm{T}+\mathrm{N}+$, but their research focused on esophageal adenocarcinoma and the prognosis was related to the number of lymph nodes dissected, while our study focused on ESCC. Although the conclusions were similar in terms of the prognosis of positive lymph nodes, the number of lymph nodes dissected in this study was divided into $\leq 19$ or $>19$ according to a cutoff value, and seemed to be statistically significant in the univariate analysis, but there was no statistical difference in the multivariate analysis $(\mathrm{P}=0.094)$. Two other implications also arise. In relation to lymph node status, if the preoperative positive lymph nodes do not respond to NCRT, there will be a severely negative impact on long-term survival. Second, a thorough lymph node dissection may improve prognosis.

As for the recurrence and metastasis pattern of EC with pathological remission after radiotherapy and chemotherapy, Barbetta et al. (20) showed that although there was a similarity in the cumulative recurrence rate between esophageal adenocarcinoma and ESCC, the recurrence pattern seemed to be different, and ESCC tended to have local recurrence. Conflicting with Barbetta's study, our findings showed that, ESCC recurrence more commonly manifested as distant metastasis.

This study demonstrated a linear correlation between the cumulative ESCC mortality after NCRT in primary 
remission and the rate of recurrence and metastasis, which is a pattern that occurs with other solid tumors. A prognosis of postoperative lymph node positive is poor, which makes more prone to recurrence and metastasis. Xi et al. (21) found a similar conclusion with esophageal adenocarcinoma.

This study may provide a basis for certain clinical decisions. To limit the presence of positive postoperative pathological lymph nodes, we believe that (I) It is very important to accurately evaluate lymph node status prior to the initiation of treatment; for example, in addition to enhanced CT, PET-CT, endoscopic ultrasound biopsy and ultrasound bronchoscopy biopsy can be combined according to the situation. (II) For the positive lymph nodes before treatment, local treatment should be strengthened, or combined with immunotherapy, so as to maximize the pathological remission rate of lymph nodes and primary lesions.

This study has some limitations. First, it is a retrospective study with a small sample size. Secondly, a small number of cases that were not included due to incomplete follow-up information might have produced a degree of statistical bias.

\section{Conclusions}

This study revealed that an independent risk factor for survival prognosis in ESCC patients with ypT0 after NCRT followed by surgery was the presence of a positive postoperative pathological lymph nodes, which shortened survival time of this group of patients via increased recurrence and metastasis.

\section{Acknowledgments}

We would like to thank all the members of for the Department of Cardiothoracic Surgery and the Department of Radiotherapy in our hospital who participated in this research.

Funding: None.

\section{Footnote}

Reporting Checklist: The authors have completed the STROBE reporting checklist. Available at http://dx.doi. org/10.21037/atm-20-4864

Data Sharing Statement: Available at http://dx.doi. org/10.21037/atm-20-4864

Conflicts of Interest: All authors have completed the ICMJE uniform disclosure form (available at http://dx.doi. org/10.21037/atm-20-4864). The authors have no conflicts of interest to declare.

Ethical Statement: The authors are accountable for all aspects of the work in ensuring that questions related to the accuracy or integrity of any part of the work are appropriately investigated and resolved. All procedures performed in this study involving human participants were in accordance with the Declaration of Helsinki (as revised in 2013). This study was approved by the ethics committee of Enze Hospital of the Taizhou Enze Medical Center (group) in Zhejiang province, China. As this was a retrospective study, informed consent was not required.

Open Access Statement: This is an Open Access article distributed in accordance with the Creative Commons Attribution-NonCommercial-NoDerivs 4.0 International License (CC BY-NC-ND 4.0), which permits the noncommercial replication and distribution of the article with the strict proviso that no changes or edits are made and the original work is properly cited (including links to both the formal publication through the relevant DOI and the license). See: https://creativecommons.org/licenses/by-nc-nd/4.0/.

\section{References}

1. Torre LA, Bray F, Siegel RL, et al. Global cancer statistics, 2012. CA Cancer J Clin 2015;65:87-108.

2. Ma WJ, Zhang QN, Shi SZ, et al. Preoperative chemoradiation may be more effective for esophageal squamous cell carcinoma compared with adenocarcinoma: results from 15 randomized controlled trials of 2,250 patients. Transl Cancer Res 2018;7:1421-30.

3. Arnold M, Soerjomataram I, Ferlay J, et al. Global incidence of oesophageal cancer by histological subtype in 2012. Gut 2015;64:381-7.

4. Shang QX, Yang YS, Hu WP, et al. Prognostic significance and role of thoracic lymph node metastasis based on Chinese expert consensus in esophageal cancer. Ann Transl Med 2019;7:381.

5. Park IH, Kim JY. Surveillance or resection after chemoradiation in esophageal cancer. Ann Transl Med 2018;6:82.

6. Yang H, Liu H, Chen Y, et al. Neoadjuvant Chemoradiotherapy Followed by Surgery Versus Surgery Alone for Locally Advanced Squamous Cell Carcinoma of the Esophagus (NEOCRTEC5010): A Phase III 
Multicenter, Randomized, Open-Label Clinical Trial. J

Clin Oncol 2018;36:2796-803.

7. Wan T, Zhang XF, Liang C, et al. The Prognostic Value of a Pathologic Complete Response After Neoadjuvant Therapy for Digestive Cancer: Systematic Review and Meta-Analysis of 21 Studies. Ann Surg Oncol 2019;26:1412-20.

8. van Hagen P, Wijnhoven BP, Nafteux P, et al. Recurrence pattern in patients with a pathologically complete response after neoadjuvant chemoradiotherapy and surgery for oesophageal cancer. Br J Surg 2013;100:267-73.

9. Soror T, Kho G, Zhao KL, et al. Impact of pathological complete response following neoadjuvant chemoradiotherapy in esophageal cancer. J Thorac Dis 2018;10:4069-76.

10. Eyck BM, van der Wilk BJ, Lagarde SM, et al. Neoadjuvant chemoradiotherapy for resectable oesophageal cancer. Best Pract Res Clin Gastroenterol 2018;36-37:37-44.

11. Hamai Y, Hihara J, Emi M, et al. Evaluation of Prognostic Factors for Esophageal Squamous Cell Carcinoma Treated with Neoadjuvant Chemoradiotherapy Followed by Surgery. World J Surg 2018;42:1496-505.

12. Rice TW, Blackstone EH, Rusch VW. 7 th edition of the AJCC Cancer Staging Manual: esophagus and esophagogastric junction. Ann Surg Oncol 2010;17:1721-4.

13. Chen B, Zhang B, Zhu C, et al. Modified McKeown minimally invasive esophagectomy for esophageal cancer: a 5 -year retrospective study of 142 patients in a single institution. PLoS One 2013;8:e82428.

14. Owaki T, Matsumoto M, Okumura H, et al. Endoscopic ultrasonography is useful for monitoring the tumor response of neoadjuvant chemoradiation therapy in esophageal squamous cell carcinoma. Am J Surg

Cite this article as: Kong M, Shen J, Zhou C, Yang H, Chen B, Zhu C, Wang G. Prognostic factors for survival in esophageal squamous cell carcinoma (ESCC) patients with a complete regression of the primary tumor (ypT0) after neoadjuvant chemoradiotherapy (NCRT) followed by surgery. Ann Transl Med 2020;8(18):1129. doi: 10.21037/atm-20-4864
2012;203:191-7.

15. Depypere L, Moons J, Lerut T, et al. Neoadjuvant chemoradiation treatment followed by surgery for esophageal cancer: there is much more than the mandard tumor regression score. Acta chirurgica Belgica 2016;116:149-55.

16. Rice TW, Ishwaran H, Kelsen DP, et al. Recommendations for neoadjuvant pathologic staging (ypTNM) of cancer of the esophagus and esophagogastric junction for the 8th edition AJCC/UICC staging manuals. Dis Esophagus 2016;29:906-12.

17. Shapiro J, van Lanschot JJB, Hulshof M, et al. Neoadjuvant chemoradiotherapy plus surgery versus surgery alone for oesophageal or junctional cancer (CROSS): long-term results of a randomised controlled trial. Lancet Oncol 2015;16:1090-8.

18. Blum Murphy M, Xiao L, Patel VR, et al. Pathological complete response in patients with esophageal cancer after the trimodality approach: The association with baseline variables and survival-The University of Texas MD Anderson Cancer Center experience. Cancer 2017;123:4106-13.

19. Depypere LP, Vervloet G, Lerut T, et al. ypT0N+: the unusual patient with pathological complete tumor response but with residual lymph node disease after neoadjuvant chemoradiation for esophageal cancer, what's up? J Thorac Dis 2018;10:2771-8.

20. Barbetta A, Sihag S, Nobel T, et al. Patterns and risk of recurrence in patients with esophageal cancer with a pathologic complete response after chemoradiotherapy followed by surgery. J Thorac Cardiovasc Surg 2018;S0022-5223(18)33122-2.

21. Xi M, Hallemeier CL, Merrell KW, et al. Recurrence Risk Stratification After Preoperative Chemoradiation of Esophageal Adenocarcinoma. Ann Surg 2018;268:289-95. 